\title{
OPTIMALITY OF SIZE-WIDTH TRADEOFFS FOR RESOLUTION
}

\author{
Maria Luisa Bonet and Nicola Galesi
}

\begin{abstract}
This paper is concerned with the complexity of proofs and of searching for proofs in resolution. We show that the recently proposed algorithm of Ben-Sasson \& Wigderson for searching for proofs in resolution cannot give better than weakly exponential performance. This is a consequence of our main result: we show the optimality of the general relationship called size-width tradeoff in Ben-Sasson \& Wigderson. Moreover we obtain the optimality of the size-width tradeoff for the widely used restrictions of resolution: regular, Davis-Putnam, negative, positive.
\end{abstract}

Keywords. Resolution, Complexity of Proofs, Automated Theorem Proving

Subject classification. 03F20, 68T15, 03B05

\section{Introduction}

Proof Complexity Theory is concerned with proving non-trivial lower bounds on the length of proofs of classes of tautologies in sound and complete propositional proof systems. This question is closely related to an important open problem in complexity theory. Cook \& Reckhow (1979) proved that $N P \neq c o-N P$ iff for every propositional proof system there is a class of tautologies that require superpolynomial size proofs. Proving superpolynomial lower bounds is also very relevant to the study of automated theorem provers. In many applications, given a possible tautology, we are faced with the problem of finding a proof of it, if one exists. Then we encounter two problems: (1) the complexity of the smallest possible proof, which might be exponential in the size of the tautology, and (2) the complexity of the proof search.

Regarding the first problem, most probably no propositional proof system can prove all tautologies efficiently, otherwise $P$ would be equal to $N P$ (Cook \& Reckhow 1979), which is generally believed to be false. One approach to fixing the inherent inefficiency of propositional proof systems, is to use the more efficient ones. Then we are faced with the second problem. How hard is it then to find proofs? It seems that the more efficient a proof system is, the harder it is 
to find proofs in it. Bonet et al. (2000b) defined a notion of automatizability. A propositional proof system is automatizable if and only if there is a deterministic procedure to find proofs in that system in polynomial time with respect to the smallest proof in that system. In the sequence of papers Krajǐcek \& Pudlàk (1998) and Bonet et al. $(1998,2000 b)$ it is proved that any propositional proof system that simulates bounded-depth Frege is not automatizable, unless some widely accepted cryptographic conjectures are violated. There is also evidence that resolution is not automatizable (see Alekhnovich \& Razborov (2001)).

There are some algorithms to find proofs in some proof systems. For instance, Davis et al. (1962), Ben-Sasson \& Wigderson (2001) and Beame \& Pitassi (1996) gave algorithms for resolution, and Clegg et al. (1996) for polynomial calculus. The algorithms of Ben-Sasson \& Wigderson (2001) and Beame \& Pitassi (1996) are both weakly exponential for resolution, and that of Clegg et al. (1996) is polynomial for the system of polynomial calculus with bounded polynomial degree. Therefore this bounded-degree polynomial calculus is automatizable. In this paper we study the performance of the algorithm proposed in Ben-Sasson \& Wigderson (2001) for finding resolution refutations.

Ben-Sasson \& Wigderson (2001), based on previous work of Haken (1985) and Beame \& Pitassi (1996), defined the width as a complexity measure for resolution refutations. Width of a refutation is the maximal number of literals in any clause of the refutation. The importance of considering this measure is twofold. On one side they were able to give a general relationship between the width and the length of a refutation, reducing the problem of giving lower bounds on the length to that of giving lower bounds on the width. The widthsize relation can be stated as follows: If $F$, an unsatisfiable formula over $n$ variables, has a resolution refutation of size $S$, then it has a resolution refutation of width $O(\sqrt{n \log S})$. Through this relationship they obtained a unified method to prove most of the previously known lower bounds for resolution. On the other side Ben-Sasson \& Wigderson (2001) made explicit a new simple proof-search algorithm based on searching for clauses of increasing size. This algorithm works in time $T(n)=n^{O(w)}$ where $w$ is the minimal width of any refutation of $F$.

The tradeoff of Ben-Sasson \& Wigderson (2001) shows that for $k$-CNF, with $k$ a constant, if we can give a width lower bound equal to the number of variables, then we have an exponential lower bound for the size of refuting the formula. An immediate interesting question arising from the work of BenSasson \& Wigderson (2001) is whether we can improve the size-width tradeoff there obtained. That is, can we get a weaker width lower bound (e.g. to the square root of the number of variables), but still obtain an exponential lower 
bound for the size?

Notice that an affirmative answer to this question for the case of unrestricted resolution, could give exponential lower bounds for the size of resolution refutations of formulas such as the $P H P_{n}^{m}, m \geq n^{2}$ which at this point use a more complicated argument (see Raz 2002; Razborov 2002).

Here we give a negative answer to this question for the case of unrestricted resolution. In fact we find a $k-C N F$ formula built over $O\left(n^{2}\right)$ variables, $M G T_{n}$, having polynomial size unrestricted resolution refutations, but requiring $\Omega(n)$ (the square root of the number of variables) width to be refuted. Combining our result with the size-width tradeoff for tree-like resolution it turns out that $M G T_{n}$ requires exponential size tree-like resolution proofs, and therefore it provides an exponential separation between tree-like and unrestricted resolution (see also Ben-Sasson \& Wigderson 2001; Bonet et al. 2000a).

In this paper we also study whether for restrictions of resolution, different from tree-like, we can obtain a better tradeoff result then that given for unrestricted resolution. We give a negative answer to this question. For restrictions of resolution such as regular, Davis-Putnam, positive and negative, we show the optimality of the same size-width tradeoff given for unrestricted resolution. As for the case of unrestricted resolution this fact implies an exponential separation between tree-like resolution and all the restrictions considered. Similar results were obtained in Bonet et al. (2000a) and Ben-Sasson et al. (2001).

Another important, even if negative, consequence of our result is that the proof search algorithm proposed by Ben-Sasson \& Wigderson (2001) is not going to be efficient for finding resolution refutations in any of the restrictions of resolution we consider.

In Section 2 we give the preliminary definitions that will be needed throughout the paper. In Section 3 we show the optimality of the width-size method. In Section 4 we prove some consequences of the results of section 3.

\section{Preliminaries}

Let $\mathcal{V}=\left\{x_{1}, \ldots, x_{n}\right\}$ be a set of boolean variables. For a variable $x_{i} \in \mathcal{V}$ let $\bar{x}_{i}$ denote the negation of $x_{i}$. A literal $\ell_{i}$ can be either a variable $x_{i}$ or its negation, with the convention that if $\ell_{i}=\bar{x}_{i}$, then $\bar{\ell}_{i}=x_{i}$ and that $\overline{\bar{x}}_{i}=x_{i}$. A clause is a disjunction of literals $\left\{\ell_{i_{1}}, \ldots, \ell_{i_{k}}\right\}$, possibly empty. A C(onjuntive) $\mathrm{N}$ (ormal) $\mathrm{F}$ (orm) formula is a conjunction of clauses. For a formula $F$, let $\operatorname{Lit}(F)$ be the set of literals of $F$. 
Resolution is a refutation proof system for formulas in CNF form based on the following resolution rule:

$$
\frac{C \vee x \quad \bar{x} \vee D}{C \vee D}
$$

where if $C$ and $D$ have common literals, they appear only once in $C \vee D$. A resolution refutation of a $\mathrm{CNF}$ formula $F$ is a derivation of the empty clause from the clauses defining $F$, using the above inference rule.

We also consider some restrictions of the resolution proof system.

o tree-like resolution. Any clause in the proof is used at most once as premise in a resolution rule.

- regular resolution. The proofs are restricted in such a way that any variable can be eliminated at most once in any path from an initial clause to the empty clause.

- Davis-Putnam resolution. The proofs are restricted in such a way that there exists an ordering of the variables such that if a variable $x$ is eliminated before a variable $y$ on any path from an initial clause to the empty clause, then $x$ is before $y$ in the ordering. Notice that Davis-Putnam resolution is necessarily regular.

- negative resolution. The application of the resolution rule is restricted to the case in which one of the premises must not contain any positive literals.

- positive resolution. The application of the resolution rule is restricted to the case in which one of the premises must not contain any negative literals.

Let $R \vdash F$ denote that $R$ is a refutation of $F$ (we use the notation $\vdash_{t l}$ to denote that the proof is tree-like). The size $|R|$ of a refutation $R$ is the number of clauses used in $R$. The size complexity $S(\vdash F)$ of refuting a $C N F$ formula $F$ in resolution, is defined as $\min _{R \vdash F}|R|$.

Following Ben-Sasson \& Wigderson (2001) the width $w(F)$ of a $C N F$ formula $F$ is defined to be the the number of literals of the largest clauses in $F$. The width $w(R)$ of a refutation $R$ is defined as the size of the greatest clause appearing in $R$. The width $w(\vdash F)$ of refuting a formula $F$ is defined as $\min _{R \vdash F} w(R)$. 
The following theorem, proved in Ben-Sasson \& Wigderson (2001), gives the tradeoff between size and width for the tree-like and the dag-like resolution systems:

Theorem 2.1 (Ben-Sasson \& Wigderson 2001). Let $F$ be any unsatisfiable CNF formula. Then:

(i) $S\left(\vdash_{t l} F\right) \geq 2^{(w(\vdash F)-w(F))}$;

(ii) $S(\vdash F) \geq \exp \left(c \cdot \frac{(w(\vdash F)-w(F))^{2}}{|\operatorname{Lit}(F)|}\right)$,

where the constant $c$ is an absolute constant, independent of $F$.

In what follows we will define the graph tautology that we will use to prove the optimality of the size-width tradeoff. A binary relation $R$ is asymmetric if and only if for all pairs $(x, y),(x, y) \in R \rightarrow(y, x) \notin R$ and is linear if for all $(x, y)$, either $(x, y) \in R$ or $(y, x) \in R$. A (strict) ordering is a transitive and asymmetric relation. A linear (strict) ordering is a strict order that is also linear.

It is straightforward to note that any strict order over a finite set defines (at least) a minimal element. When we consider the directed graph associated to a strict order, the previous property is equivalent to the following: each directed acyclic graph, closed under transitivity and without loops must have a source node.

We define a $C N F$ formula, $G T_{n}$, expressing the negation of the previous property. $\forall i, j \in[n], i \neq j$, let $x_{i, j}$ be a variable whose intended meaning is that $(i, j)$ is a directed edge in a graph over $[n] . G T_{n}$ is then defined by the following clauses over $n(n-1)$ variables:

$$
\begin{array}{lll}
\text { (1) } & \bar{x}_{i, j} \vee \bar{x}_{j, k} \vee x_{i, k} & i, j, k \in[n], i \neq j, j \neq k, k \neq i \\
\text { (2) } \bar{x}_{i, j} \vee \bar{x}_{j, i} & i, j \in[n], i \neq j \\
\text { (3) } \bigvee_{k=1, k \neq j}^{n} x_{k, j} & j \in[n]
\end{array}
$$

where the clauses in (1) say that the graph is transitive, those in (2) that it is asymmetric and those in (3) that there is no source node.

Krishnamurthy (1985) was the first to consider this formula and to study its complexity for resolution refutations. He conjectured that $G T_{n}$ required long proofs in resolution. Stalmark (1996) refuted Krishnamurthy's conjecture giving polynomial size unrestricted resolution refutations. 


\section{Optimality of the size-width tradeoff}

Consider the equation giving the size-width tradeoff for the case of unrestricted resolution:

$$
S(\vdash F) \geq \exp \left(c \cdot \frac{(w(\vdash F)-w(F))^{2}}{|\operatorname{Lit}(F)|}\right),
$$

where $c$ is an absolute constant independent of the formula $F$.

One way to show that this tradeoff is (almost) optimal is to find an infinite family of unsatisfiable $C N F$ formulas $F_{n}$ such that

○ $w\left(F_{n}\right)$ is $O(1)$

○ $S\left(\vdash F_{n}\right)$ is $n^{O(1)}$

$\circ w\left(\vdash F_{n}\right)$ is $\Omega\left(\sqrt{\left|\operatorname{Lit}\left(F_{n}\right)\right|}\right)$

○ $\left|\operatorname{Lit}\left(F_{n}\right)\right|$ goes to infinity as $n$ grows.

Note that when $F$ fulfills the three previous conditions, the tradeoff formula doesn't give us an exponential lower bound.

We will consider a modification of the formula $G T_{n}$, that we call $M G T_{n}$, and we show that $M G T_{n}$ fulfills the above requirements. We will show (see Theorem 3.2) that polynomial size resolution refutations for $M G T_{n}$ can be easily obtained from polynomial size resolution refutations of $G T_{n}$. Therefore we start by giving a polynomial size resolution refutation for $G T_{n}$. This was first given by Stalmark (1996). Our proof slightly differs from that of Stalmark. However, this difference allows us to show that our refutations respect the further restrictions of being Davis-Putnam (and therefore regular) and positive.

THEOREM 3.1. There are polynomial size refutations of $G T_{n}$ in the following proof systems: (i) dag-like resolution, (ii) Davis-Putnam resolution, (iii) regular resolution, (iv) positive resolution.

Proof. We start by giving a scheme of the proof. Consider the following abbreviations:

$$
\begin{aligned}
& C_{m}(j):=\bigvee_{i=1, i \neq j}^{m} x_{i, j} \text { for all } j \in[n] \\
& A(i, j, k):=\bar{x}_{i, j} \vee \bar{x}_{j, k} \vee x_{i, k} \text { for all } i, j, k \in[n] i \neq j \neq k \neq i \\
& B(i, j):=\left(\bar{x}_{i, j} \vee \bar{x}_{j, i}\right) \text { for all } i, j \in[n] i \neq j
\end{aligned}
$$


The idea of the proof is to obtain clauses of the form $C_{m}(j)$ from $m=n$ down to $m=2$ in the following way:

$$
\begin{array}{lllll}
C_{n}(1) & C_{n}(2) & \ldots & C_{n}(n-1) & C_{n}(n) \\
C_{n-1}(1) & C_{n-1}(2) & \ldots & C_{n-1}(n-1) & \\
\vdots & & & & \\
C_{2}(1) & C_{2}(2) & &
\end{array}
$$

For each $k, C_{k}(1), \ldots, C_{k}(k)$ are obtained in parallel, and for $j=1, \ldots, k-1$, each $C_{k-1}(j)$ is obtained using the clauses $C_{k}(j)$ and $C_{k}(k)$ derived in the previous step, and the initial clauses $A(1, k, j), A(2, k, j), \ldots, A(k-1, k, j)$ and $B(j, k)$. At the end we easily derive the empty clause from $C_{2}(1), C_{2}(2)$ and $B(2,1)$. Now we provide more details of the proof and show that it respects the various restrictions of resolution.

Consider the following abbreviations:

$$
\begin{array}{ll}
D_{k-1}^{j}(i):=C_{k-1}(j) \vee \bar{x}_{i, k} & k \in[n] /\{1\}, i \in[k-1], j \in[n] \\
E_{k-1}^{j}(i):=\left(C_{k-1}(j) \vee \bigvee_{\ell=i}^{k-1} x_{\ell, k}\right) & k \in[n] /\{1\}, i \in[k-1], j \in[n]
\end{array}
$$

The proof proceeds by steps going from $m=n$ to $m=2$. All the clauses $C_{n}(j)$, for all $j \in[n]$, are initial clauses and therefore derivable. At the $n-k+1$ th step, for each $j=1, \ldots, k-1$, we prove in parallel each of the $C_{k-1}(j)$ in the following way:

(a) Perform in parallel the following resolution steps, each one resolving the variable $x_{k, j}$ :

$$
\begin{array}{ll}
(1) & \frac{C_{k}(j) A(1, k, j)}{D_{k-1}^{j}(1)} \\
(2) & \frac{C_{k}(j) A(2, k, j)}{D_{k-1}^{j}(2)} \\
\vdots & \\
(j-1) & \frac{C_{k}(j) \quad A(j-1, k, j)}{D_{k-1}^{j}(j-1)} \\
(j) & \frac{C_{k}(j) B(j, k)}{D_{k-1}^{j}(j)} \\
(j+1) & \frac{C_{k}(j) A(j+1, k, j)}{D_{k-1}^{j}(j+1)} \\
\vdots & \\
(k-1) & \frac{C_{k}(j) A(k-1, k, j)}{D_{k-1}^{j}(k-1)}
\end{array}
$$

(b) $C_{k-1}(j)$ is then obtained by the following (tree-like) refutation in which we are resolving on the variables $x_{1, k}, x_{2, k}, \ldots, x_{k-1, k}$ : 


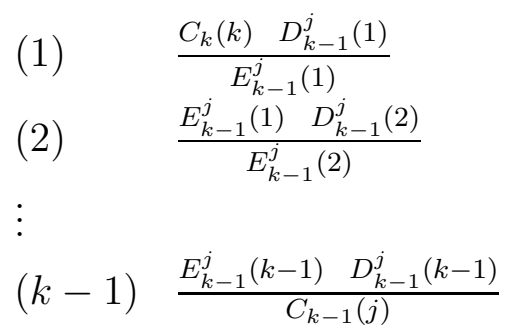

Such a refutation respects the positive restriction, since at each resolution step one of the premises contains only positive literals. It is also easy to see that the following order of elimination of the variables is respected:

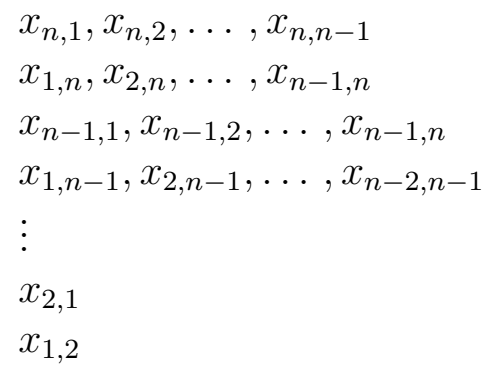

Therefore the refutation is Davis-Putnam as well as regular.

In the above refutation there are clauses of size $\Omega(n)$. We show below that in fact any refutation of $G T_{n}$ must have clauses of such width. Note however that in $G T_{n}$ there are initial clauses of size $n-1$ and therefore $G T_{n}$ does not fulfill the first requirement needed to show the optimality of the size-width tradeoff. We consider a modified version of $G T_{n}, M G T_{n}$, such that: (1) $w\left(M G T_{n}\right) \leq 3$; (2) $\left|\operatorname{Lit}\left(M G T_{n}\right)\right|=2 n^{2}-n$; (3) from a refutation of $G T_{n}$ we can easily find a refutation of $M G T_{n}$, and (4) $w\left(\vdash M G T_{n}\right) \geq \Omega(n)$.

Consider the clauses with large width in the definition of $G T_{n}$ :

$$
\bigvee_{k=1, k \neq j}^{n} x_{k, j} \text { for } j \in[n]
$$

For each $j \in[n]$ let $y_{0, j}, \ldots y_{j-1, j} y_{j+1, j}, \ldots y_{n, j}$ be $n$ new extension variables. $M G T_{n}$ is defined by replacing the large clauses in the definition of $G T_{n}$ with the following set of clauses of constant width:

$$
\bar{y}_{0, j} \wedge \bigwedge_{i=1, i \neq j}^{n}\left(y_{i-1, j} \vee x_{i, j} \vee \bar{y}_{i, j}\right) \wedge y_{n, j} \quad \text { for all } j \in[n]
$$

The intended meaning of the $y$ variables is: 


$$
y_{i j}=\bigvee_{k=1, k \neq j}^{i} x_{k j}
$$

$M G T_{n}$ has then constant initial width, and it is defined over $2 n^{2}-n$ literals. It remains to prove that: (1) there are polynomial size resolution refutations of $M G T_{n}$ and (2) the minimal width for refuting $M G T_{n}$ is $\Omega(n)$ (observe that the number of variables in $M G T_{n}$ is $O\left(n^{2}\right)$ ). We start by proving that the upper bound holds, even for the restrictions of resolution.

THEOREM 3.2. There are polynomial size refutations for the formula $M G T_{n}$ in any of the following proof systems: (i) resolution, (ii) positive resolution, (iii) Davis-Putnam resolution, (iv) regular resolution.

Proof. The proof follows a standard argument. From the initial clauses of $M G T_{n}$

$$
\bar{y}_{0, j} \wedge \bigwedge_{i=1, i \neq j}^{n}\left(y_{i-1, j} \vee x_{i, j} \vee \bar{y}_{i, j}\right) \wedge y_{n, j} \quad \text { for all } j \in[n]
$$

we derive the initial clauses of $G T_{n}$

$$
\bigvee_{k=1, k \neq j}^{n} x_{k, j} \text { for all } j \in[n]
$$

resolving on the $y$ variables once at time and in a tree-like fashion. Then we apply the polynomial size proof of $G T_{n}$ to these new clauses using the other initial clauses. The part of the proof eliminating the $y$ variables is in fact a tree-like proof of size quadratic in $n$. Since the $y$ variables get resolved one time only, the regularity of the proof is preserved. It is also easy to see that the new first part of the proof is a Davis-Putnam resolution since the following order of elimination of the $y$ variables is respected:

$y_{0,1}, \ldots, y_{n, 1}$,

$y_{0,2}, \ldots, y_{n, 2}$,

$\vdots$

$y_{0, n}, \ldots, y_{n-1, n}$.

To obtain a positive resolution refutation we only have to be careful to eliminate the $y_{k, j}$ variables starting from $y_{n, j}$ for all $j \in[n]$. 

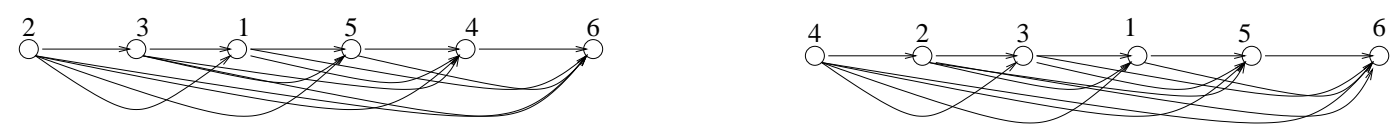

Figure 3.1: Two linear directed graphs giving a 2-cta and a 4-cta for for $G T_{6}$.

In order to prove the lower bound for the width of refuting $M G T_{n}$, we need to introduce the notion of a critical truth assignment for the formula $M G T_{n}$. We start by giving the definition of critical assignments for $G T_{n}$ and then extend it to the case of $M G T_{n}$. A linear directed graph is the graph associated to a strict linear order, i.e. a directed acyclic graph, closed under transitivity, without loops, and in which every two nodes are linked by an edge (see Figure $3.1)$.

Definition 3.3. A critical truth assignment $\alpha$ for $G T_{n}$ is a linear directed graph.

The idea is that if the variable $x_{i, j}$ correponds to the existence of a directed edge $(i, j)$ in the graph, then such a linear graph falsifies only one among the initial clauses of $G T_{n}$. This is because the graph is closed under transitivity, there are no cycles, and every node except for the first one in the line has a predecessor. We call a critical assignment a $j$-cta if $j$ is the first element in the linear graph. A $j$-cta falsifies only the initial clause

$$
\bigvee_{i \in[n], i \neq j} x_{i, j}
$$

Switching from a $j$-cta to a $k$-cta is very easy in terms of the linear graph. We put the node $k$ in the first position in the line, and move all nodes before $k$ one position forward. In terms of the adjacency matrix of the graph this means changing the 1's by 0 's in column $k$ and the 0 by 1 's in row $k$. The following matrices show how to obtain a 4-cta from a 2-cta for $G T_{6}$ (see also Figure 3.1).

\begin{tabular}{cccccc}
\multicolumn{4}{c}{ 2-cta for } & $G T_{6}$ \\
$*$ & 0 & 0 & $\mathbf{1}$ & 1 & 1 \\
1 & $*$ & 1 & $\mathbf{1}$ & 1 & 1 \\
1 & 0 & $*$ & $\mathbf{1}$ & 1 & 1 \\
$\mathbf{0}$ & $\mathbf{0}$ & $\mathbf{0}$ & $*$ & $\mathbf{0}$ & 1 \\
0 & 0 & 0 & $\mathbf{1}$ & $*$ & 1 \\
0 & 0 & 0 & 0 & 0 & $*$
\end{tabular}

4-cta for $G T_{6}$

* $0 \begin{array}{lllll}0 & 0 & \mathbf{0} & 1 & 1\end{array}$

$1 * 1 \quad 0011$

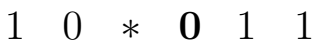

$1 \quad 1 \quad 1 \quad * \quad 1 \quad 1$

$0 \quad 0 \quad 0 \quad 00 * 1$

$\begin{array}{llllll}0 & 0 & 0 & 0 & 0 & *\end{array}$

Denote with $C_{j}$ the formula $\bar{y}_{0, j} \wedge \bigwedge_{i=1, i \neq j}^{n}\left(y_{i-1, j} \vee x_{i, j} \vee \bar{y}_{i, j}\right) \wedge y_{n, j}$ in the definition of $M G T_{n}$. 
Definition 3.4. A $j$-cta $\alpha$ for $M G T_{n}$ is obtained by $j$-cta for $G T_{n}$ assigning boolean values to the extension variables $y$ 's in such a way that $\alpha \forall C_{j}$ and for all $k \neq j, \alpha \models C_{k}$.

The next lemma shows that the previous definition is sound, that is we can always extend a $j$-cta for $G T_{n}$ to a $j$-cta for $M G T_{n}$.

Lemma 3.5. Any j-cta for $G T_{n}$ can be extended to a $j$-cta for $M G T_{n}$.

Proof. $\quad$ Let $\alpha$ be the $j$-cta for $G T_{n} . \beta$ will be the $j$-cta for $M G T_{n} . \alpha$ falsifies the initial clause $\bigvee_{k=1, k \neq j}^{n} x_{k, j}$. Hence it also falsifies the formula $C_{j}$ for any possible assignment to the the variables $y_{i, j}$ for $i=0, \ldots, n$. To define $\beta$ we have to specify how to assign values to the $y_{i, k}$ variables for $i=0, \ldots, n$ and for $k \neq j$, in such a way to satisfy $C_{k}$. We can do this by considering $y_{i, k}=\bigvee_{l=1, l \neq k}^{i} x_{j, k}$, and assigning the same value to $y_{i, k}$ as $\alpha$ assigns to the disjunction.

Notice that there is not a unique way to extend a $j$-cta for $G T_{n}$ to a $j$-cta for $M G T_{n}$. The variables $y_{i, k}$ don't have to take the intended meaning for the $j$-cta to satisfy $C_{k}$.

Recall that $C_{j}$ is the formula $\bar{y}_{0, j} \wedge \bigwedge_{i=1, i \neq j}^{n}\left(y_{i-1, j} \vee x_{i, j} \vee \bar{y}_{i, j}\right) \wedge y_{n, j}$.

Let $\operatorname{Vars}(j)$ be the set of variables in $C_{j}$ together with $x_{j, i}$ for all $i \neq j$; i.e. the variables $x_{i, j}, x_{j, i}$ for all $i \in[n], i \neq j$, and the variables $y_{i, j}$ for $i \in\{0\} \cup[n]$, $i \neq j$.

Lemma 3.6. Let $K$ be a clause, and $l$ the number of literals appearing in $K$. Then at most $2 \cdot l$ sets $\operatorname{Var}(j)$ have variables appearing in $K$.

Proof. $\quad$ Every variable $x_{i, j}$ belongs to two different sets, $\operatorname{Var} s(i)$ and $\operatorname{Var} s(j)$. Each variable $y_{s, i}$ belongs to one set, $\operatorname{Var} s(i)$. So each variable of $K$ can belong to at most two sets $\operatorname{Vars}(j)$.

THEOREM 3.7. Any resolution proof of $M G T_{n}$ must have a clause of width $\Omega(n)$.

Proof. For each $I \subseteq[n]$, let $C_{I}$ be defined as $\bigwedge_{i \in I} C_{i}$. For any clause $C$ in a resolution proof of $M G T_{n}$, let $I_{C}$ be a minimal $I \subseteq[n]$ such that all critical truth assignments satisfying $C_{I}$ also satisfy $C$. For any clause $C$ we define a measure $\mu(C)$ as the cardinality of $I_{C}$. Observe that for any $i \in[n], \mu\left(C_{i}\right) \leq 1$. $\mu(\{\})=n$ since $C_{I}$ is satisfiable when $I \neq[n] . \mu$ is obviously subadditive, that 
is for any step in the resolution proof, the measure of the conclusion is less than or equal to the sum of the measures of its premises. Therefore in any resolution proof of $M G T_{n}$ there is a clause, say $K$, such that $\frac{n}{3} \leq \mu(K)<\frac{2 n}{3}$. We show that this clause will contain $\geq \frac{n}{6}$ literals. Assume for the sake of contradiction that $|K|<\frac{n}{6}$. Consider the set $I_{K}$ and notice that $\left|I_{K}\right|=\mu(K) \geq \frac{n}{3}$. By Lemma 3.6 there is an $l \in I_{K}$, such that no variable from $\operatorname{Var} s(l)$ belongs to $K$. Let $l$ be such an index from $I_{K}$.

Consider any critical assignment $\alpha$ such that $\alpha\left(C_{l}\right)=0, \alpha(K)=0$ and for all $j \in I_{K} /\{l\} \alpha\left(C_{j}\right)=1$. This assignment exists by the minimality of $I_{K}$ and moreover it satisfies all the clauses $C_{i}$ for $i \in[n] /\{l\}$. Define $J=[n] / I_{K}$. Since $|J| \geq \frac{n}{3}$ (because $\left|I_{K}\right|<\frac{2 n}{3}$ ), by Lemma 3.6 we conclude that there is at least a $j \in J$ such that no variable from $\operatorname{Var} s(j)$ appears in $K$. We build a $j$-critical truth assignment $\beta$ from $\alpha$ such that $\beta\left(C_{i}\right)=1$ for all $i \in I_{K}$ and $\beta(K)=0$, and this leads to a contradiction by the definition of $I_{K}$.

$\beta$ is built by the following three steps. At the first step we change the value of the $x$ variables in $M G T_{n}$, switching the $l$-cta $\alpha$ to a $j$-cta. This first step does not affect the value of $K$ since we only change the variables $x_{i, j}$ and the variables $x_{j, i}$. These are in $\operatorname{Var} s(j)$ and no variable from $\operatorname{Var} s(j)$ appears in $K$.

For $\beta$ to be a $j$-cta it has to satisfy the formulas $C_{k}$ for $k \neq j$. Consider the case $k=l$. After the first step $\beta\left(x_{j, l}\right)=0$, hence the second step in the definition of $\beta$ will be to change the values of the variables $y_{i, l}$ for $i=0, \ldots, n$ as in Lemma 3.5. This last change will not affect the value of $K$ since for $i=0, \ldots, n$ the variables $y_{i, l}$ belong to $\operatorname{Vars}(l)$ and no variable from $\operatorname{Vars}(l)$ appears in $K$.

Finally we need $\beta$ to satisfy the formulas $C_{s}$ for all $s \neq l$ and $s \neq j$. In the third step we define $\beta\left(y_{i, s}\right)=\alpha\left(y_{i, s}\right)$ for $s \neq l$ and $s \neq j$. Notice that, no $x$ variable is changed from 1 to 0 , except for those belonging to the $j$-th column of the assignment (see e.g. previous example). Therefore, since $\alpha\left(C_{s}\right)=1$ for all $s \neq l$ and $s \neq j$, then we have $\beta\left(C_{s}\right)=1$ for all $s \neq l$ and $s \neq j$.

Under the hypothesis that $|K|<n / 6$, we have defined an assignment $\beta$ that falsifies $K$, and satisfies all $C_{i}$, for $i \in I_{K}$. This is a contradiction by the definition of $I_{K}$. Therefore, $|K| \geq n / 6$.

The following is an immediate corollary of previous two theorems.

THEOREM 3.8. There is a family of constant-width $C N F$ formulas $\left\{F_{n}\right\}$ on $O\left(n^{2}\right)$ variables verifying the following two properties: (1) $\left\{F_{n}\right\}$ has polynomial 
size resolution refutations; (2) any resolution refutation of $\left\{F_{n}\right\}$ contains a clause having width at least $\Omega(n)$.

\section{Consequences of the optimality result}

Our result has several consequences. First of all the size-width tradeoff of BenSasson \& Wigderson (2001) for tree-like resolution together with Theorem 3.7 give a lower bound of $2^{\Omega(n)}$ for the size of tree-like resolution proofs of $M G T_{n}$.

TheOREm 4.1. Any tree-like resolution proof of $M G T_{n}$ must have size $\Omega\left(2^{n}\right)$.

This Theorem allows us to prove that tree-like resolution is exponentially separated from the other restricted systems of resolution we consider. These separations were only recently obtained in Bonet et al. (2000a) and Ben-Sasson et al. (2001).

THEOREM 4.2. Tree-like resolution is exponentially separated from unrestricted, positive, negative, regular and Davis-Putnam.

Proof. For the case of regular, Davis-Putnam, positive and unrestricted resolution, the result is immediate since $M G T_{n}$ has polynomial size refutation in these systems, but, by the previous theorem, require exponential size tree-like refutations. For the case of negative resolution, we consider the unsatisfiable formula $\overline{M G T}_{n}$ in which the $x_{i, j}$ variables are replaced by $z_{i, j}$ whose intended meaning is opposite to that of the $x$ variables. It is easy to see that the positive resolution proof for $M G T_{n}$ is in fact a negative resolution proof for $\overline{M G T}_{n}$. Moreover the technique to obtain the lower bound for the width can also be applied. We leave to the reader the details of this fact. This means that the shortest tree-like resolution refutations of $\overline{M G T}_{n}$ are exponentially long in $n$. And therefore tree-like resolution is exponentially separated also from negative resolution.

As we have seen in Section 2, the size-width tradeoff for tree-like resolution is much stronger than that of dag-like resolution. This fact, combined with our optimality result for the size-width tradeoff for dag-like resolution, allows us to obtain an exponential separation between tree-like and dag-like resolution (Theorem 4.2). Therefore an interesting question is to know whether we can also obtain exponential separations between other restrictions of resolution (e.g. regular) and resolution, showing better tradeoffs for such restrictions of 
resolution. Our next theorem implies that this is not the case for regular, positive, negative and Davis-Putnam resolution. In fact we prove the optimallity of the size-width tradeoff for all these restrictions.

THEOREM 4.3. The tradeoff

$$
S(\vdash F) \geq \exp \left(c \cdot \frac{(w(\vdash F)-w(F))^{2}}{|\operatorname{Lit}(F)|}\right)
$$

is optimal for the systems of regular, positive, negative and Davis-Putnam resolution. The constant $c$ is again an absolute constant.

Proof. The polynomial size refutations for $M G T_{n}$ provided in Theorem 3.2 are also in all the restrictions but negative resolution. By Theorem 3.7 any resolution refutation of $M G T_{n}$ (in particular in any of the considered restrictions) must have a clause of size $\Omega(n)$. The result is hence immediate for these restrictions. In the case of negative resolution the result follows by an argument similar to that applied in the previous Theorem.

A refutation system is automatizable if there is an algorithm $\mathcal{A}$ such that, for any unsatisfiable formula $F, \mathcal{A}$ finds a refutation of $F$ in that system in time polynomial in the size of the shortest proof of $F$ in that system.

Ben-Sasson \& Wigderson (2001) considered a simple algorithm, for finding resolution proofs based on the idea of seeking for refutations of minimal width. Let $F$ be a unsatisfiable $C N F$ formula. Consider the following algorithm:

$\mathrm{C}:=$ Clauses of $F$

$w:=0$

While $\square \notin C$ Do

$w:=w+1$

apply resolution rule to clauses in $C$ to derive

all possible clauses of width $\leq w$.

Add the clauses obtained to $C$

End

By definition of width it is immediate to see that the possible number of clauses that the above algorithm can produce is bounded by $n^{O(w(\vdash F))}$. Therefore the running time of the algorithm on input $F$ is bounded by $n^{O(w(\vdash F))}$. Our main result (Theorem 3.8), implies that it cannot be used to obtain the automatizability of any of the resolution systems considered. This is because 
we have a formula, $M G T_{n}$, that has polynomial size resolution refutations, but, since the minimal width for refuting $M G T_{n}$ is $\Omega(n)$, then the algorithm will require an exponential number of steps to find a refutation of $M G T_{n}$ in any of the considered restrictions.

\section{Acknowledgements}

Maria Luisa Bonet was partly supported by Project CICYT, TIC 98-0410-C0201, Promoción General del Conocimiento PB98-0937-C04-03 and TIC20001970-CE. Part of this work was done while Nicola Galesi was a member of the School of Mathematics at the Institute for Advanced Study (Princeton) supported by a NSF grant CCR-9987845. The authors would like to thank the anonymous referees for many comments about the preliminary version of this paper which appeared in Bonet \& Galesi (1999). These comments contributed to improve the quality of the presentation.

\section{References}

M. Alekhnovich \& A. Razborov (2001). Resolution is not Automatizable unless $\mathrm{W}[\mathrm{P}]$ is Tractable. In Proceedings of the IEEE Symposium on Foundations of Computer Science (FOCS), 190-199. To appear.

P. Beame \& T. Pitassi (1996). Simplified an Improved Resolution Lower Bounds. In Proceedings of the IEEE Symposium on Foundations of Computer Science (FOCS), 274-282.

E. Ben-Sasson, R. Impagliazzo \& A. Wigderson (2001). Near Optimal Separations of General and Tree-like Resolution. Submitted. Appeared in the ECCC as TR00-005.

E. Ben-SAsson \& A. Wigderson (2001). Short Proofs are Narrow - Resolution made simple. Journal of the Association for the Computing Machinery 48(2), 149168. A preliminary version appeared in the Proceedings of the 1999 Annual ACM Symposium on Theory of Computing.

M. Bonet, C. Domingo, R. Gavaldá, A. Maciel \& T. Pitassi (1998). Nonautomatizability of Bounded-Depth Frege Proofs. In Proceedings of the IEEE Conference on Computational Complexity, 15-23. To appear in Journal of Computational Complexity.

M.L. Bonet, J.L. Esteban, N. Galesi \& J. Johannsen (2000a). On the Relative Complexity of Resolution Refinements and Cutting Planes Proof Systems. SIAM 
Journal on Computing 30(5), 1462-1484. A preliminary version appeared in the Prooceedings of the 1999 IEEE Symposium on Foundations of Computer Science with the title Exponential Separations between Restricted Resolution and Cutting Planes Proof Systems.

M.L. Bonet \& N. Galesi (1999). A Study of Proof Search Algorithms for Resolution and Polynomial Calculus. In Proceedings of the IEEE Symposium on Foundations of Computer Science (FOCS), 422-431.

M.L. Bonet, T. Pitassi \& R. Raz (2000b). On Interpolation and Automatization for Frege Systems. SIAM Journal on Computing 29(6), 1939-1967. A preliminary version appeared in the Proceedings of the 1997 Symposium IEEE Symposium on Foundations of Computer Science with the title No Feasible interpolation for $\mathrm{TC}^{0}$ Frege proofs.

Matthew Clegg, Jeffrey Edmonds \& Russell Impagliazzo (1996). Using the Groebner Basis Algorithm to Find Proofs of Unsatisfiability. In Proceedings of the Twenty-Eighth Annual ACM Symposium on the Theory of Computing, 174-183. Philadelphia, Pennsylvania.

S. CoOK \& R. RECKHow (1979). The relative efficiency of propositional proof systems. Journal of Symbolic Logic 44, 36-50.

M. Davis, G. Logemann \& D. Loveland (1962). A machine program for theorem proving. Communication of the ACM 5, 394-297.

A. HAKen (1985). The intractability of resolution. Theoretical Computer Science 39, 297-308.

J. KRAJǏČEK \& P. PUdLÀK (1998). Some consequences of cryptographical conjectures for $S_{2}^{1}$ and EF. Information and Computation 140, 82-94. Preliminary version in D. Leivant, ed., LCC '94, Springer LNCS 960, 1995.

B. Krishnamurthy (1985). Short Proofs for Tricky Formulas. Acta informatica 22, 253-275.

R. RAZ (2002). Resolution Lower Bounds for the Weak Pigeonhole Principle. To appear in the Proceedings of the ACM Symposium on Theory of Computing (STOC).

A. Razborov (2002). Improved Resolution Lower Bounds for the Weak Pigeonhole Functional Principle. To appear in Theoretical Computer Science.

G. Stalmark (1996). Short Resolution Proofs for a Sequence of Tricky Formulas. Acta Informatica 33, 277-280. 
MARIA Luisa Bonet

Departament de Llenguatges i Sistemes Informàtics

Universitat Politècnica de Catalunya

Jordi Girona Salgado 1-3

08034 Barcelona, Spain.

bonet@lsi.upc.es
Nicola Galesi

Departament de Llenguatges i Sistemes Informàtics

Universitat Politècnica de Catalunya

Jordi Girona Salgado 1-3

008034 Barcelona, Spain.

galesi@lsi.upc.es

Department of Computer Science

University of Toronto

10 King's College Road

Toronto, Ontario

CANADA M5S 3G4

galesi@cs.toronto.edu 